Supporting information

\title{
Poorly soluble marketed drugs display solvation limited solubility
}

\author{
Christel A. S. Bergström, Carola M. Wassvik, Kajsa Johansson and Ina Hubatsch \\ Pharmaceutical Screening and Informatics, Department of Pharmacy, Uppsala \\ University, Uppsala Biomedical Centre P.O. Box 580, SE-751 23 Uppsala, Sweden.
}

Content: LC-MS/MS settings for analysis of the compounds studied; time versus solubility profiles of the compounds studied; a correlation matrix of physicochemical properties, molecular descriptors and solubility; formulation approaches to increase solubility for the compounds studied; dose regimen of the compounds; and PLS model statistics and loading plots for the final model based on poorly soluble compounds. 
Table S1. Set up of LC-MS/MS

\begin{tabular}{lllll}
\hline & Polarity & $\begin{array}{l}\text { Parent ion } \\
\text { detected }\end{array}$ & $\begin{array}{l}\text { Product ion } \\
\text { detected }\end{array}$ & Gradient $^{\mathrm{a}}$ \\
\hline \hline albendazole & + & & 234.8 & $\mathrm{C}$ \\
carvedilol & + & & 222.1 & $\mathrm{~A}$ \\
cinnarizine & + & & 168.0 & $\mathrm{~B}$ \\
danazol & + & 338.5 & 148.1 & $\mathrm{C}$ \\
felodipine & + & & 338.0 & $\mathrm{C}$ \\
glimepiride & + & & 352.5 & $\mathrm{C}$ \\
itraconazol & + & & 392.1 & $\mathrm{C}$ \\
rimonabant & + & & 363.0 & $\mathrm{C}$ \\
tamoxifen & + & 372.50 & 129.0 & $\mathrm{C}$ \\
terfenadine & + & & 436.3 & $\mathrm{C}$ \\
tolfenamic acid & - & & 216.0 & $\mathrm{D}$ \\
troglitazone & - & 440.0 & & $\mathrm{E}$ \\
\hline
\end{tabular}

${ }^{\mathrm{a}}$ Composition of gradients and mobile phases are presented in table S2

Table S2. Composition of mobile phases and gradients used in chromatography

\begin{tabular}{|c|c|c|c|c|c|c|c|}
\hline A & $\begin{array}{l}\text { Time } \\
\text { (min) }\end{array}$ & $\begin{array}{l}0.1 \% \text { FA in } \\
\text { water }(\%)\end{array}$ & $\begin{array}{l}0.1 \% \text { Fa in } \\
\text { ACN }(\%)\end{array}$ & $\mathrm{D}$ & $\begin{array}{l}\text { Time } \\
\text { (min) }\end{array}$ & $\begin{array}{l}\begin{array}{l}\text { water } \\
(\%)\end{array} \\
\end{array}$ & $\begin{array}{l}\text { ACN } \\
(\%)\end{array}$ \\
\hline & 0.00 & 70 & 30 & & 0.00 & 70 & 30 \\
\hline & 1.00 & 10 & 90 & & 1.00 & 10 & 90 \\
\hline & 3.00 & 10 & 90 & & 3.00 & 10 & 90 \\
\hline & 3.10 & 70 & 30 & & 3.10 & 70 & 30 \\
\hline & 5.00 & 70 & 30 & & 5.00 & 70 & 30 \\
\hline $\mathrm{B}$ & $\begin{array}{l}\text { Time } \\
\text { (min) }\end{array}$ & $\begin{array}{l}0.1 \% \text { FA in } \\
\text { water }(\%) \\
\end{array}$ & $\begin{array}{l}0.1 \% \text { Fa in } \\
\text { ACN (\%) }\end{array}$ & $\mathrm{E}$ & $\begin{array}{l}\text { Time } \\
\text { (min) }\end{array}$ & $\begin{array}{l}\text { water } \\
(\%)\end{array}$ & $\begin{array}{l}\text { ACN } \\
(\%)\end{array}$ \\
\hline & 0.00 & 70 & 30 & & 0.00 & 70 & 30 \\
\hline & 0.20 & 70 & 30 & & 1.00 & 10 & 90 \\
\hline & 2.10 & 10 & 90 & & 2.00 & 10 & 90 \\
\hline & 3.10 & 10 & 90 & & 2.10 & 70 & 30 \\
\hline & 3.20 & 70 & 30 & & 5.00 & 70 & 30 \\
\hline & 5.00 & 70 & 30 & & & & \\
\hline C & $\begin{array}{l}\text { Time } \\
\text { (min) }\end{array}$ & $\begin{array}{l}0.1 \% \text { FA in } \\
\text { water }(\%)\end{array}$ & $\begin{array}{l}0.1 \% \mathrm{Fa} \text { in } \\
\text { ACN (\%) }\end{array}$ & & & & \\
\hline & 0.00 & 70 & 30 & & & & \\
\hline & 1.00 & 10 & 90 & & & & \\
\hline & 2.00 & 10 & 90 & & & & \\
\hline & 2.10 & 70 & 30 & & & & \\
\hline & 5.00 & 70 & 30 & & & & \\
\hline
\end{tabular}


Table S3. Correlation matrix (R).

\begin{tabular}{|c|c|c|c|c|c|c|c|c|c|c|c|c|c|}
\hline & $\log S$ & $\Delta \mathrm{S}_{\mathrm{m}}$ & $\Delta \mathrm{H}_{\mathrm{m}}$ & $\mathrm{T}_{\mathrm{m}}$ & Mw & $\mathrm{Clog} P$ & Polarisability & Rot bonds & NPSA & PSA & $\mathrm{HB}_{\text {tot }}$ & $\mathrm{HB}_{\mathrm{a}}$ & $\mathrm{HB}_{\mathrm{d}}$ \\
\hline $\mathrm{HB}_{\mathrm{d}}$ & 0.37 & 0.19 & 0.25 & 0.33 & -0.10 & -0.65 & -0.18 & 0.03 & -0.33 & 0.71 & 0.54 & 0.24 & 1.00 \\
\hline $\mathrm{HB}_{\mathrm{a}}$ & -0.11 & 0.31 & 0.37 & 0.23 & 0.77 & -0.16 & 0.54 & 0.51 & 0.39 & 0.78 & 0.95 & 1.00 & \\
\hline $\mathrm{HB}_{\text {tot }}$ & 0.03 & 0.34 & 0.40 & 0.31 & 0.63 & -0.35 & 0.41 & 0.44 & 0.23 & 0.94 & 1.00 & & \\
\hline PSA & 0.17 & 0.13 & 0.23 & 0.43 & 0.38 & -0.54 & 0.16 & 0.14 & -0.03 & 1.00 & & & \\
\hline NPSA & $-0.48^{a}$ & 0.09 & 0.03 & -0.28 & 0.87 & 0.58 & 0.94 & 0.80 & 1.00 & & & & \\
\hline Rot bonds & -0.53 & 0.38 & 0.29 & -0.42 & 0.81 & 0.40 & 0.86 & 1.00 & & & & & \\
\hline Polarisability & $-0.61^{a}$ & 0.11 & 0.07 & -0.16 & 0.93 & 0.45 & 1.00 & & & & & & \\
\hline ClogP & -0.71 & -0.24 & -0.32 & -0.43 & 0.36 & 1.00 & & & & & & & \\
\hline Mw & $-0.48^{\mathrm{a}}$ & 0.12 & 0.11 & -0.07 & 1.00 & & & & & & & & \\
\hline $\mathrm{T}_{\mathrm{m}}$ & 0.13 & -0.13 & 0.09 & 1.00 & & & & & & & & & \\
\hline$\Delta \mathrm{H}_{\mathrm{m}}$ & 0.09 & 0.97 & 1.00 & & & & & & & & & & \\
\hline$\Delta \mathrm{S}_{\mathrm{m}}$ & 0.07 & 1.00 & & & & & & & & & & & \\
\hline
\end{tabular}

Troglitazone was not included in the correlations since this compound was determined qualitatively $(<15.9 \mathrm{nM})$. The following

abbreviations are used: $\log S=\log _{10}$ of intrinsic solubility $(\mathrm{M}) ; \Delta \mathrm{S}_{\mathrm{m}}=$ entropy of melting $(\mathrm{J} / \mathrm{mol} \times \mathrm{K}) ; \Delta \mathrm{H}_{\mathrm{m}}=$ enthalpy of melting $(\mathrm{kJ} / \mathrm{mol})$;

$\mathrm{Mw}=$ =molecular weight; $\mathrm{Clog} \mathrm{P}=$ Calculated octanol/water partition coefficient; Rot bonds=number of rotatable bonds; NPSA=non-polar

surface area; PSA=polar surface area; $\mathrm{HB}_{\text {tot }}=$ total number of hydrogen bond acceptors and donors; $\mathrm{HB}_{\mathrm{a}}=$ number of hydrogen bond acceptors;

$\mathrm{HB}_{\mathrm{d}}=$ number of hydrogen bond donors.

a) The correlation between the size related descriptors Mw, polarisability and NPSA increased to $-0.61,-0.79$ and -0.85 , respectively after exclusion of outlier

tolfenamic acid. 
Table S4. Composition of formulations of poorly soluble compound

\begin{tabular}{|l|c|c|c|c|c|c|c|c|c|c|c|c|c|c|c|}
\hline & 1 & 2 & 3 & 4 & 5 & 6 & 7 & 8 & 9 & 10 & 11 & 12 & 13 & 14 & 15 \\
\hline Albendazole & & & $\bullet$ & & & & & $\bullet$ & & $\bullet$ & & & & $\bullet$ & \\
\hline Astemizole & & $\bullet$ & & & & & & & $\bullet$ & & $\bullet$ & $\bullet$ & & & $\bullet$ \\
\hline Carvedilol & & $\bullet$ & & & & & & & $\bullet$ & & $\bullet$ & & & & \\
\hline Cinnarizine & & & & & & & & & $\bullet$ & & $\bullet$ & & & & $\bullet$ \\
\hline Danazol & & & & & & & & & & & & & & & $\bullet$ \\
\hline Felodipine & & & & & & & & & $\bullet$ & & $\bullet$ & & $\bullet$ & & \\
\hline Glimepiride & & & & & & & & & & & $\bullet$ & & & & $\bullet$ \\
\hline Glyburide & & $\bullet$ & & & $\bullet$ & & & & & & & & & & \\
\hline Indomethacin & & $\bullet$ & & & & & & & & & & & & $\bullet$ & $\bullet$ \\
\hline Itraconazol & & & & $\bullet$ & & & & & & & & & & & $\bullet$ \\
\hline Rimonabant & & & & & & & & & & & & & & & $\bullet$ \\
\hline Tamoxifen & $\bullet$ & $\bullet$ & & $\bullet$ & & $\bullet$ & & & & & & & $\bullet$ & & $\bullet$ \\
\hline Terfenadine & $\bullet$ & & $\bullet$ & & $\bullet$ & & & $\bullet$ & $\bullet$ & $\bullet$ & & & & \\
\hline Tolfenamic acid & & & & & & \\
\hline Troglitazone &
\end{tabular}

Excipients included in the formulations of importance for disintegration, dissolution and solubility are numbered as follow below. Information regarding the formulations was obtained from PDR, FASS (Swedish PDR), product specifications and the web. Differences can be found in the formulations for compounds supplied by several companies. The action of each additive was obtained from The Handbook of Pharmaceutical Excipients, The Pharmaceutical Press, London, 1994.

\section{Alginic acid}

Stabilizing agent, tablet and capsule disintegrant, tablet binder, viscosity-increasing agent.

\section{Colloidal silicon dioxide}

Adsorbent; anticaking agent; glidant; suspending agent; tablet disintegrant; viscosityincreasing agent

\begin{tabular}{ll}
\hline Use & C (\%) \\
\hline Emulsion stabilizer & $1-5$ \\
Suspending and thickening agent & $2-10$ \\
Glidant & $0.1-0.5$ \\
\hline
\end{tabular}

\section{Carmellos sodium/Carboxymethylcellulosum natricum}

Coating agent, tablet and capsule disintegrant, tablet binder, stabilizing agent, suspending agent, viscosity-increasing agent.

\section{Croscarmellose sodium}

Tablet (0.5-5.0\%) and capsule (10-25\%) disintegrant.

\section{Lecithin}

Emollient, emulsifying agent, solubilizing agent. 


\section{Macrogol/Polyethylene glycol}

Tablet lubricant. Can also be used to enhance solubility and dissolutionby making solid dispersion.

\section{Macrogol stearate}

Emulsifying agent; solubilizing agent; wetting agent.

\section{Magnesium alumina silicate}

A polymer complex of $\mathrm{Al}, \mathrm{Mg}$, silicon, $\mathrm{O}$ and $\mathrm{H}_{2} \mathrm{O}$. $\mathrm{Al}$ is substituted to varying degrees by Mg.

\begin{tabular}{ll}
\hline Use & C (\%) \\
\hline Adsorbent & $10-50$ \\
Binding agent & $2-10$ \\
Disintegrating agent & $2-10$ \\
Emulsion stabilizer (oral) & $1-5$ \\
Suspending agent (oral) & $0.5-2.5$ \\
Stabilizing agent & $0.5-2.5$ \\
Viscosity modifier & $2-10$ \\
\hline
\end{tabular}

\section{Microcrystalline cellulose}

Adsorbent, suspending agent, tablet and capsule diluent, tablet disintegrant.

\begin{tabular}{ll}
\hline Use & C (\%) \\
\hline Adsorbent & $20-90$ \\
Antiadherent & $5-20$ \\
Disintegrating agent & $5-15$ \\
Capsule diluent & $20-90$ \\
Tablet diluent & $20-90$ \\
\hline
\end{tabular}

\section{Polysorbate $80 / 90$}

Emulsifying agent, nonionic surfactant, solubilizing agent, wetting agent.

\begin{tabular}{ll}
\hline Use & C (\%) \\
\hline Emulsifying agent in o/w & $1-15$ \\
Solubilizing agent in lipophilic bases & $1-10$ \\
Wetting agent in lipophilic bases & $0.1-3$ \\
\hline
\end{tabular}

11. Polyvinylpyrrolidon/Vinylacetate polymers/Povidon/Crospovidon Suspending agent, tablet binder.

\begin{tabular}{ll}
\hline Use & C (\%) \\
\hline Carrier for drugs & $10-25$ \\
Dispersing/suspending agent & Up to 5 \\
Tablet binder/diluent or coating & $0.5-5$ \\
\hline
\end{tabular}

The solubility of a number of poorly soluble compounds may be increased by mixing with polyvinylpyrrolidon/povidon. 


\section{Sodium lauryl sulphate (E487)}

Anionic surfactant, detergent, emulsifying agent, skin penetrant, tablet and capsule lubricant, wetting agent.

\begin{tabular}{ll}
\hline Use & C (\%) \\
\hline Anionic emulsifier & $0.5-2.0$ \\
Solubilizer in C>CMC & $>0.0025$ \\
Tablet lubricant & $1-2$ \\
Wetting agent & $1-2$ \\
\hline
\end{tabular}

\section{Sodium starch glycolate (type A)/Sodium carboxymethyl starch}

Tablet and capsule disintegrant.

\section{Sorbitan laurate/Sorbitan stearate/Sorbitan ester}

Emulsifying agent, non-ionic surfactant, solubilizing agent, wetting agent.

\begin{tabular}{ll}
\hline Use & $\mathbf{C ~ ( \% )}$ \\
\hline Emulsifying agent & $1-15$ \\
Solubilizing agent & $1-10$ \\
Wetting agent & $0.1-3$ \\
\hline
\end{tabular}

\section{Starch}

Binder, diluent, disintegrant (3-15\% w/w). 
Table S5. Dose Regimen

\begin{tabular}{|c|c|c|c|c|}
\hline & Dose (mg) & Dose interval & Oral formulation & Other \\
\hline Albendazole $^{\mathrm{a}}$ & n.a. & n.a. & Oral suspension & Veterinary medicine \\
\hline Astemizole $^{\mathrm{b}}$ & 10 & 24 & Tablet & Food intake reduces absorption with $60 \%$ \\
\hline Carvedilol $^{\mathrm{a}}$ & $3.1 / 6.3 / 12.5 / 25$ & $12 / 24 h$ & Tablet & Food intake delays $\mathrm{C}_{\max }$. \\
\hline Cinnarizine $^{\mathrm{a}}$ & 10 & 8/ 12/ 24h & Tablet & No information on food effects \\
\hline Danazol $^{\mathrm{b}}$ & $50 / 100 / 200$ & $12 \mathrm{~h}$ & Capsule & No information on food effects \\
\hline Felodipine $^{\mathrm{a}}$ & $2.5 / 5.0 / 10$ & $24 \mathrm{~h}$ & Tablet (CR) & Absorption rate increases in fed state. \\
\hline Glimepiride $^{\mathrm{a}}$ & $1 / 2 / 3 / 4$ & $24 \mathrm{~h}$ & Tablet & Complete absorption; no significant effect of food. \\
\hline Glyburide $^{\mathrm{a}}$ & $1.75 / 3.5$ & $12 / 24 h$ & Tablet & Complete absorption; no significant effect of food. \\
\hline Indomethacin $^{\mathrm{a}}$ & $25 / 50$ & $4-6 h$ & Hard capsule & Complete absorption; no information on food effects. \\
\hline Itraconazol $^{\mathrm{a}}$ & $100 / 200$ & $12 / 24 h$ & Hard capsule & Oral bioavailability increases in fed state. \\
\hline Rimonabant $^{\mathrm{a}}$ & 20 & $24 \mathrm{~h}$ & Tablet & Oral bioavailability increases in fed state. \\
\hline Tamoxifen $^{\mathrm{a}}$ & $10 / 20 / 30 / 40$ & $24 \mathrm{~h}$ & Tablet & Rapid absorption; no information on food effects. \\
\hline Terfenadine $^{c}$ & n.a. & n.a. & n.a. & n.a. \\
\hline Tolfenamic acid ${ }^{\mathrm{d}}$ & 200 & n.a. & Tablet & No information on food effects \\
\hline Troglitazone $^{\mathrm{c}}$ & n.a. & n.a. & n.a. & n.a. \\
\hline \multicolumn{5}{|c|}{$\begin{array}{l}\text { Not applicable is abbreviated n.a.; Controlled release is abbreviated CR. } \\
\text { aa Information was taken from www.fass.se, the Swedish PDR. } \\
\text { b Information was taken from PDR. } \\
\text { cThe compound has been withdrawn from the market. }\end{array}$} \\
\hline
\end{tabular}



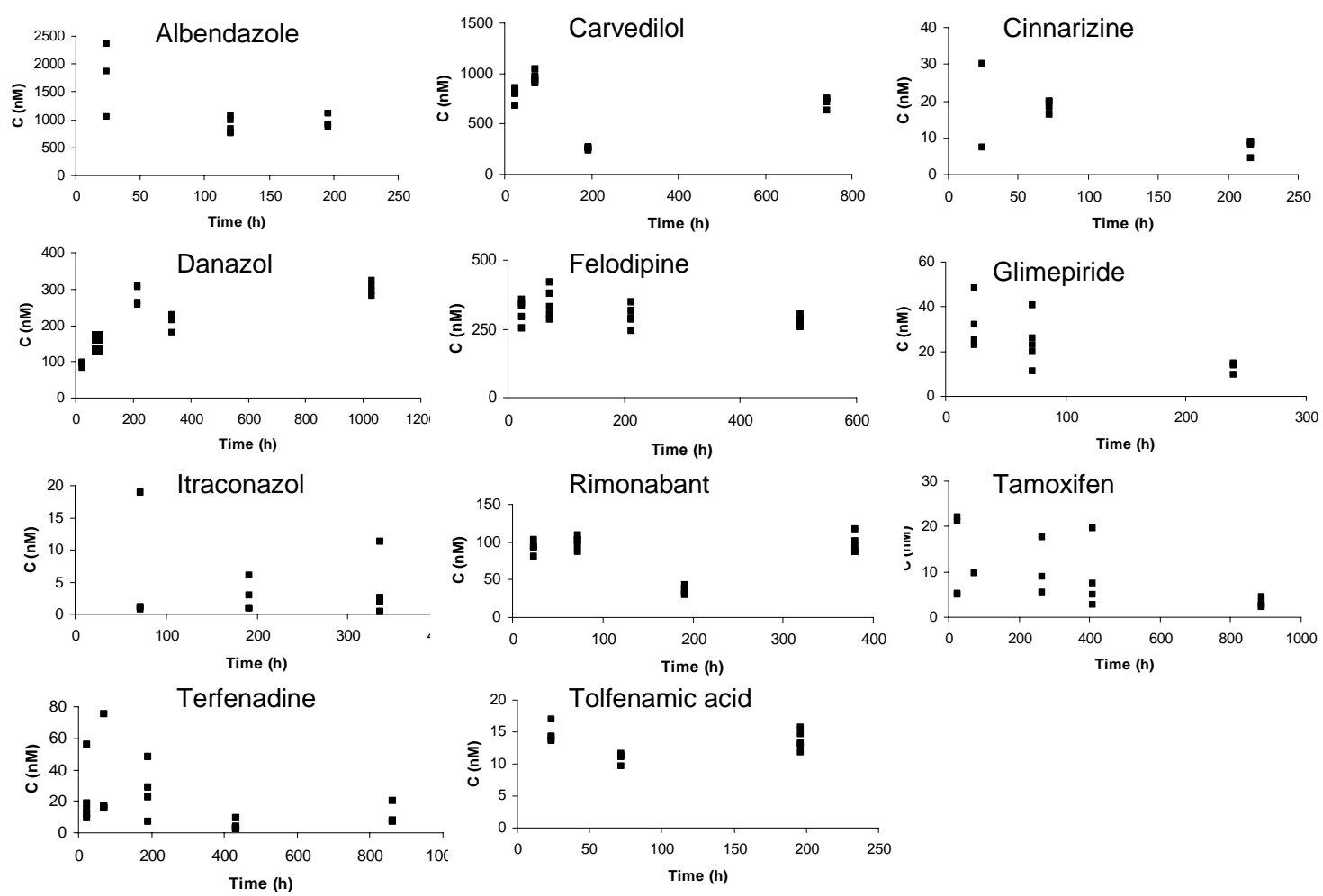

Figure S1. Time solubility profiles of the compounds measured. Cinnarizine was unstable at later time points that $72 \mathrm{~h}$, and therefore the $72 \mathrm{~h}$ solubility value is reported in table S1. Solubility data for astemizole, glyburide and indomethacin were taken from Wassvik et al, 2006, reference 7 in the paper, and therefore not included in the timesolubility study. 

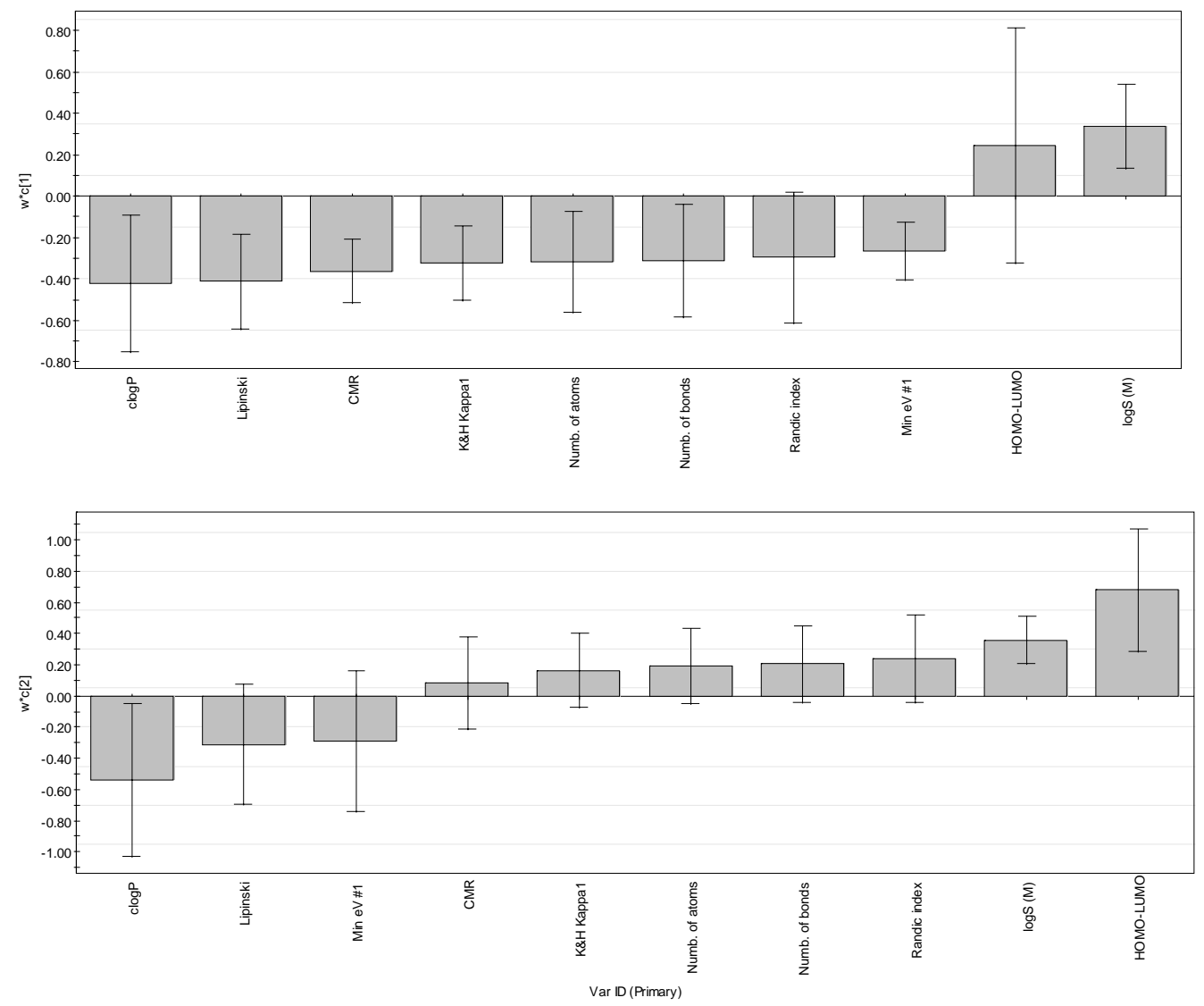

Figure S2. Loading plots of PLS analysis of physicochemical properties of importance for poor solubility. The following were identified:

Lipophilicity: ClogP

Size: Number of atoms, Number of bonds, CMR (molecular refractivity: related to size and polarisability)

Electronic distribution: Randic index (valence electrons), Kier\&Hall Kappa1 (topological index), energy difference between highest occupied and lowest unoccupied molecular orbital (HOMO-LUMO; N.B. this descriptor has negative values and hence is negatively correlated to solubility)

Miscellaneous: Min eV\#1=the smallest eigenvalue of the graph adjacency matrix from BCUT parameters. Off-diagonal elements are formal bond orders, diagonal elements are atomic weights. The descriptor is related to a low degree of aromaticity of the molecule.; Lipinski=Lipinski rule-of-five indicator.

Model statistics for the final model: $\mathrm{R}^{2}$ of 0.77 and $\mathrm{Q}^{2}$ of 0.67 (using 7 cross validation groups, e.g. leaving two compounds out at the time). The PLS model was developed to interpret which molecular properties that were of importance for the poor solubility obtained and to understand the solubility process on a mechanistic level, and not for prediction purposes. 